\title{
Narrative review: prostate-specific membrane antigen-radioligand therapy in metastatic castration-resistant prostate cancer
}

\author{
Lukas Lunger ${ }^{1}$, Robert Tauber ${ }^{1}$, Benedikt Feuerecker ${ }^{2}$, Jürgen E. Gschwend ${ }^{1}$, Matthias Eiber ${ }^{2}$, \\ Matthias M. Heck ${ }^{1}$ \\ ${ }^{1}$ Department of Urology, Rechts der Isar Medical Center, Technical University of Munich, Technical University of Munich, Munich, Germany; \\ ${ }^{2}$ Department of Nuclear Medicine, Rechts der Isar Medical Center, Technical University of Munich, Munich, Germany \\ Contributions: (I) Conception and design: L Lunger, MM Heck; (II) Administrative support: MM Heck, R Tauber, JE Gschwend; (III) Provision \\ of study materials or patients: L Lunger, MM Heck, M Eiber, B Feuerecker; (IV) Collection and assembly of data: None; (V) Data analysis and \\ interpretation: None; (VI) Manuscript writing: All authors; (VII) Final approval of manuscript: All authors. \\ Correspondence to: PD Dr. med. Matthias M. Heck. Assistant Professor, Fellow of the European Board of Urology (FEBU), Department of Urology, \\ Rechts der Isar Medical Center, Technical University of Munich (TUM), Ismaninger Str. 22, 81675 Munich, Germany. Email: matthias.heck@tum.de.
}

\begin{abstract}
Radioactive-labelled ligands targeting the prostate-specific membrane antigen (PSMA), a transmembrane protein overexpressed in prostate cancer (PC), have shown promising activity in treatment of metastatic castration-resistant prostate cancer (mCRPC). PSMA-617 and PSMA-I\&T (imaging and therapy), both labeled to the beta-emitter lutetium-177 (Lu177), are most frequently used in clinical routine and have shown a favorable side-effect profile. Common side effects are transient xerostomia. Severe side effects, e.g., treatment-associated myelosuppression, are rare. Currently treatment with Lu177-PSMA outside clinical trials is available for compassionate use for patients who exhausted conventional therapies. Previous retro- and prospective studies reported promising results with $\geq 50 \%$ PSA declines observed in at least one third of patients. Retrospective data suggests worse biochemical response in patients with visceral metastases. Preliminary data from the randomized phase II (TheraP) trial showed an improved biochemical response rate of Lu177-PSMA as compared to cabazitaxel in patients progressing after docetaxel. Following these promising data, the results of the randomized, prospective phase III VISION study are eagerly anticipated. A major challenge remains resistance to radioligand therapy with Lu177-PSMA. As an alternative, a PSMA-ligand labeled to the alpha-emitter Actinium-225 (Ac-225) may be offered to patients, which shows promising activity in patients developing progression under Lu177-PSMA at the cost of higher toxicity. Mostly permanent xerostomia is a relevant side effect resulting in treatment discontinuation in up to a quarter of patients. This review summarizes the literature on activity and toxicity of PSMA-targeted radioligand therapy in $\mathrm{mCRPC}$.
\end{abstract}

Keywords: Actinium; lutetium; prostate cancer (PC); radioligand therapy; metastatic castration-resistant prostate cancer (mCRPC); prostate-specific membrane antigen (PSMA)

Submitted Jul 31, 2020. Accepted for publication Oct 15, 2020.

doi: $10.21037 /$ tau-20-1135

View this article at: http://dx.doi.org/10.21037/tau-20-1135

\section{Introduction}

Prostate cancer (PC) is the most common malignant tumor in men and the second most common cause of cancerassociated mortality (1). In patients treated for metastatic $\mathrm{PC}$ the progression from a castration-sensitive to a castration-resistant stage marks the transition to the lethal phenotype of the disease [metastatic castration-resistant PC (mCRPC)]. In recent years, several new agents have been approved for treatment of mCRPC. They include androgen receptor-targeted therapies with abiraterone and enzalutamide as well as taxane-based chemotherapy 
with docetaxel or cabazitaxel or the bone-targeting agent radium-223-dichloride. Despite these innovations for mCRPC therapy more than 250,000 men still die of PC worldwide each year and its treatment remains challenging. Therefore, the development of novel therapeutic regimens exhibiting both effective antitumor activity and a tolerable side effect profile is warranted.

\section{Tumor therapy using prostate-specific membrane antigen (PSMA)-targeted radionuclides}

PSMA is a transmembrane protein over-expressed in PC cells in comparison to benign prostatic tissue (2). Most importantly, PSMA expression progressively increases in higher grade cancers, metastatic disease and castrationresistant PC $(2,3)$. PSMA has a catalytic site located in its extracellular domain resulting in its internalization after ligand binding (3).

At present, two PSMA ligands are reported to be most frequently used for radio-ligand therapy (RLT): PSMA-617 and PSMA-I\&T (imaging and therapy), both labelled to the low-energetic beta-emitter Lutetium-177 (4,5). Lu-177PSMA-617 was first characterized by the Heidelberg group after initial experience with I-131-MIP 1095 (6,7). Lu-177PSMA-I\&T (imaging and therapy) was first reported at the Technical University of Munich and also demonstrated nanomolar affinity for PSMA (8). To date, biodistribution of both ligands seem to have similar properties, albeit a head-to-head comparison has not yet been performed. For ease of reading, both ligands are henceforth summarized as Lu177-PSMA.

Organs at risk for a critical radiation dose upon treatment with Lu177-PSMA include the salivary glands and the kidneys (9). Review of available literature suggests that the radiation dose to bone marrow, spleen and liver are below critical limits (10).

As an alternative a PSMA-ligand labeled to the highenergetic alpha-emitter Actinium-225 (Ac-225) may be offered to patients presenting with progressive mCRPC. To date, available date suggests that RLT using Ac-225PSMA-617 may still be active in patients progressing under Lu177-PSMA. However, treatment-related permanent xerostomia remains a relevant side effect, frequently leading to discontinuation of treatment $(11,12)$. We present the following article in accordance with the Narrative Review reporting checklist (available at http://dx.doi.org/10.21037/ tau-20-1135).

\section{Methods}

This narrative review comprehensively summarizes the current knowledge on efficacy and safety of both Lu177PSMA but also Ac255-PSMA RLT for routine clinical practice. Suitable, accepted literature on Lu177- and Ac225 PSMA in PC, published and retrievable on PubMed and GoogleScholar from 2014 until August 30, 2020 was reviewed. Moreover, abstract data published at the ASCO2020 was reviewed and, if applicable, included in the review. An outlook on ongoing and future trials is given at the end of the discussion.

\section{Protocol for Lu177-PSMA RLT}

To date, Lu177-PSMA RLT is offered to mCRPC patients within a compassionate use program as a salvage therapy after having exhausted conventional therapies or within clinical trials owing to the fact that this treatment regimen has not been approved, yet (13-16). The following course of treatment is currently used in daily routine:

(I) Confirmed, sufficient PSMA expression of detected metastases proven by PSMA-directed positronemission tomography/computed tomography (PET/CT). Based on the study by Hofman et al. the minimum SUVmax of metastases ought to be at least 1.5 times the SUVmean of the liver to ensure sufficient PSMA expression in potential Lu177PSMA RLT patients (13). The EANM guidelines recommend at baseline an additional FDG-PET/ CT to exclude patients with FDG-positive, but PSMA-negative PC metastases $(15,17)$.

(II) RLT using Lu177-PSMA is offered at nuclear medicine centers. Following slow intravenous application of the radionuclide (slow injection over $10-15 \mathrm{~min}$ ) a post-therapeutic scintigraphy is performed on days 1 or 2 after treatment to evaluate in-vivo distribution of the radionuclide. Patients are usually admitted to the hospital for 2-4 days under radiation-controlled conditions. The target radiation activity for Lu177-PSMA is 4-8 giga-becquerel and is determined on individual basis.

(III) Assuming treatment response, patients typically receive Lu177-PSMA every 6 weeks for up to 4-8 cycles.

(IV) Restaging is most frequently performed after every second cycle of treatment as proposed by the EANM guidelines (15). While some institutions 
perform restaging using conventional imaging with $\mathrm{CT}$ and bone scan, others use molecular imaging with PSMA/CT. It has to be noted that tumor progression on imaging using PSMA-PET/CT may be detected earlier than with conventional imaging leading to shorter treatment with PSMARLT.

\section{Anti-tumor effect of Lu177-PSMA RLT}

The first studies investigating the activity of Lu177-PSMA RLT were retrospective and characterized by small sample sizes in mCRPC patients who exhausted conventional systemic treatments, thus representing collectives at advanced stages of the disease. Overall, the majority of these first, but also later studies reported promising results with $\geq 50 \%$ PSA declines observed in at least one third of patients. Table 1 illustrates an overview of selected retroand prospective studies available to date.

In a large German multicenter retrospective analysis involving 148 patients treated with Lu177-PSMA-617, a $\geq 50 \%$ PSA decline was defined as biochemical treatment response and was observed in $45 \%$ of patients (1). Likewise, a meta-analysis of 17 studies involving a total of 681 mCRPC patients undergoing Lu177-PSMA RLT reported a $\geq 50 \%$ PSA decline in roughly $45 \%$ of the patients (27). Similarly, data collected at our center at the Technical University Munich showed a $\geq 50 \%$ reduction of PSA serum levels in 38 of 100 analyzed patients under Lu177PSMA-I\&T following a median of 3 approved treatment regimens, underlining the promising activity of Lu177PSMA in late-stage mCRPC $(4,5)$. A subsequent subgroup analysis by clinically relevant factors revealed a significantly worse biochemical response in patients with visceral metastases. Moreover, considering progression-free survival (PFS) and overall survival (OS), both visceral metastases as well as increased serum lactate dehydrogenase (LDH) concentrations were significant independent predictors of worse treatment outcome (5). In patients with visceral metastasis median OS was significantly lower as compared to patients without evidence for visceral metastasis (7.6 versus 14.0 months, respectively). Similarly, PFS was significantly shorter for patients with visceral metastasis as compared to patients without (3.9 versus 5.9 months, respectively). Moreover, patients whose PSA concentrations achieved a decline $\geq 50 \%$ within the first 12 weeks under Lu177-PSMA RLT had a significantly improved median PFS (8.1 versus 0.4 months) (5).
The majority of available studies on Lu177-PSMA RLT investigated its activity in cohorts of mCRPC patients beyond the second or third line of conventional treatment. To further investigate the role of pretreatment with chemotherapy in mCRPC patients undergoing Lu177-PSMA RLT, Bayer et al. retrospectively analyzed 167 patients who underwent PSMA-RLT and stratified the cohort by taxane-pretreatment status (24). Of note, taxane-naive patients receiving Lu177-PSMA RLT had a significantly longer median radiographic PFS than taxanepretreated patients ( 8.8 versus 6.0 months), suggesting that patients may benefit from providing Lu177-PSMA RLT to $\mathrm{mCRPC}$ patients at an earlier time during the course of disease (24). Similarly, taxane-naive patients had a longer OS as compared to taxane-pretreated patients (27.1 versus 10.7 months, respectively).

A systematic review by von Eyben $e t$ al. investigated the activity of Lu177-PSMA RLT in comparison to conventional third line systemic treatments (abiraterone, enzalutamide or cabazitaxel). The results of this study showed a significantly better biochemical response in patients treated with Lu177-PSMA RLT as compared to other third line options. Overall, $43 \%$ of the patients treated with Lu177-PSMA RLT showed a $\geq 50 \%$ PSA decline, whereas only $21 \%$ of patients receiving conventional third line treatments achieved a PSA decline $\geq 50 \%$ (28). These results may further support a future earlier treatment algorithm role of Lu177-PSMA RLT not only as last or third line option.

In 2018, the first prospective single-arm Phase II study confirmed the activity of Lu177-PSMA RLT in $30 \mathrm{mCRPC}$ patients pretreated with at least one taxanebased chemotherapy and/or androgen receptor target therapies (abiraterone, enzalutamide). The findings of this study revealed a $\geq 50 \%$ PSA decline in $57 \%$ of the patients as well as a significant improvement in their quality of life (13). More recently, in 2020, Violet et al. published the long-term outcomes of this same cohort including a 20 -patient extension. The authors reported a median OS of 13.3 months with a statistically significant longer OS of 18.4 months in patients who had a PSA decline $\geq 50 \%$ (22). Novel data presented at the ASCO 2020 of a post-hoc analysis including 43 patients with progressive mCRPC in the RESIST phase II trial confirmed a significantly improved longer PFS (13.4 versus 3.3 months) and OS (20.1 versus 11.6 months) in patients who had a PSA decline $\geq 50 \%$ (29).

At ASCO 2020 the Hofman group presented preliminary 


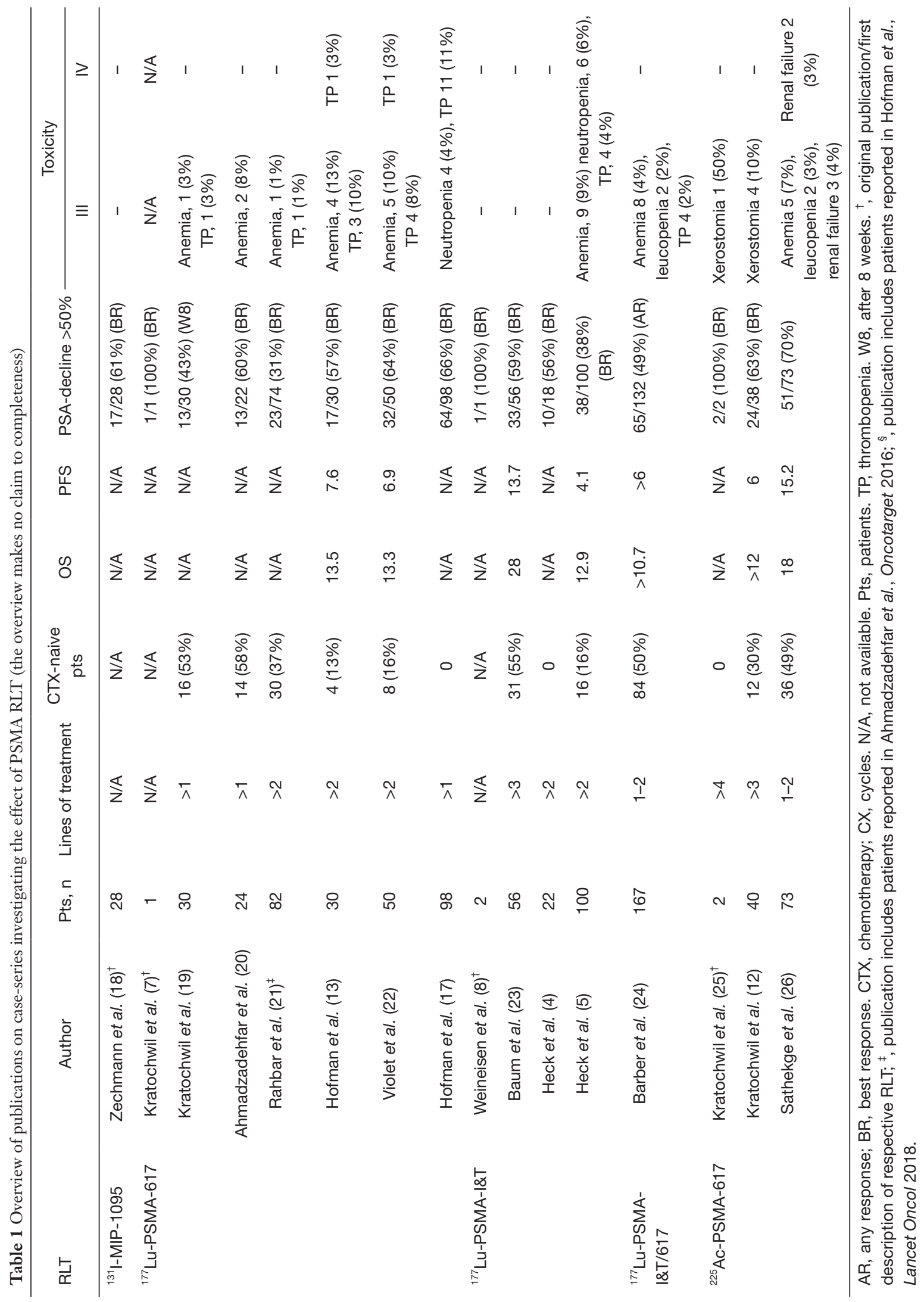


results of a first randomized Phase-2-trial (TheraP), which evaluates Lu177-PSMA versus cabazitaxel in 200 men with mCRPC upon progression to docetaxel (17). The reported trial results showed an improved biochemical response rate of Lu177-PSMA as compared to cabazitaxel in patients progressing after docetaxel (PSA decline $\geq 50 \%$ achieved in $66 \%$ versus $37 \%$ ). At a median follow-up of 13 months, treatment with Lu177-PSMA significantly improved biochemical PFS as compared to cabazitaxel (HR 0.69, 95\% CI: 0.5-0.95) (17). Data on stronger secondary endpoints like PFS and OS data are still pending. It has to be noted that the biochemical response rate in the Lu177-PSMA arm was higher compared to data from retrospective trials. One explanation might be the patient selection. In addition to a PSMA-PET/CT confirming PSMA-expression in PC metastases, an FDG-PET/CT was performed at baseline to exclude FDG-positive/PSMA-negative PC metastases. With this additional imaging procedure $28 \%$ of mCRPC patients were excluded from the trial due to PSMA-negative PC metastases.

An ongoing focus of current research is focusing on improving response rates. Preliminary, promising results of a prospective phase-I/II dose escalation trial were presented at the ASCO 2020. The data showed that fractionated dosing up to $22.2 \mathrm{GBq}$ in a population unselected for PSMA expression in a single cycle was safe in this cohort of 44 mCRPC patients with a $>50 \%$ PSA decline observed in $66.7 \%$ of patients and a median OS of 16 months (30). Also, the use of radiosensitizers to increase response rates is currently being investigated. Emmett et al presented updated interim data of the LuPIN trial to investigate the safety and efficacy of a combination of Lu177-PSMA with idronoxil (NOX66) at ASCO 2020. The PSA responses $>50 \%$ in $62 \%$ of $32 \mathrm{mCRPC}$ patients and a median survival of 17.1 months are encouraging and warrant further trials to investigate the role of both fractionated dosing and the use of radiosensitizers to increase treatment responses (31).

\section{Re-challenge using Lu177-PSMA RLT}

Of interest, the abovementioned study by Violet $\mathrm{et}$ al. also investigated the effect of additional cycles of Lu177-PSMA in comparison to other systemic treatments at relapse to Lu177-PSMA RLT as a secondary endpoint (22). In this study, patients who had shown an initial response to Lu177-PSMA RLT as defined by a PSA decline $\geq 50 \%$ and subsequently progressed in the treatment-free interval after
PSMA-RLT were considered for additional Lu177-PSMA cycles ("Re-challenge"). In this re-challenge setting 11 of 15 patients $(73 \%)$ still had a PSA decline $\geq 50 \%$ with a median OS of 26.6 months. However, although a considerable number of patients responded to re-challenge, the response to Lu177-PSMA RLT in a re-challenge setting seemed less durable with a trend for a shorter PFS as compared to the PFS observed upon initial Lu177-PSMA RLT (no direct, statistical comparison was reported). Data collected at our center at the Technical University Munich showed a PSA decline $\geq 50 \%$ in 3 of 8 patients (37.5\%) who had Lu177PSMA RLT re-challenge with a median biochemical PFS and OS of 3.2 and 14.0 months, respectively (32).

These findings suggest that a re-challenge with Lu177PSMA might be a valuable treatment option in patients who had an excellent prior response to Lu177-PSMA and subsequently progressed following a treatment pause.

\section{Safety of Lu177-PSMA RLT}

Treatment with Lu177-PSMA is generally well-tolerated. However, primary safety concerns are the physiologic expression of PSMA in salivary glands, kidneys and treatment-associated myelosuppression.

Low degree Xerostomia due to radiation toxicity are typically transient, occur within the first 1-2 weeks following infusion of Lu177 PSMA and are seen in roughly $15-24 \%$ of the patients $(5,27,33)$. Other common, nonhematological side-effects may be fatigue and nausea, seen in roughly $25 \%$ and $10 \%$, respectively. The incidence of treatment-associated nephrotoxicity is low (9.5\%) and usually rather mild (27). Severe grade 3-4 hematologic toxicity was observed in less than $10 \%$ in our retrospective study cohort (5). Similarly, the prospective phase II trial by Hofman et al. reported a relatively low frequency of grade 3-4 thrombocytopenia and anemia (17\% and 23\%, respectively) (13). Notably, it appears that treatment associated hematological toxicity primarily occurs in patients with baseline anemia/thrombocytopenia due to reduced bone marrow reserve following previous chemotherapy or metastatic osseous infiltration (13). Systematic reviews by von Eyben et al. and Emmett et al. underlined these observations and reported mild grade 1-2 anemia or thrombocytopenia in $10-25 \%$ of patients with metastatic bone marrow infiltration at baseline whereas no hematologic toxicity was observed in patients without baseline bone marrow damage $(28,33)$. 


\section{RLT with Actinium-255-PSMA-617}

Despite its favorable toxicity and promising activity in previously published studies, roughly one fourth of patients have shown resistance to Lu177-PSMA RLT despite confirmed sufficient PSMA expression (27). An alternative or salvage treatment approach upon progression under Lu177-PSMA is RLT with Actinium-255 PSMA-617 (AcPSMA). Contrary to the beta particle emitter Lu177, treatment with Ac-255 takes advantage of the shorter tissue penetration range, higher linear energy transfer and favorable microdosimetry provided by the alpha particles. The use of the alpha-emitter Ac255 labelled to PSMA-617 was first described by Kratochwil et al. in 2016 (25). In this study, two patients were treated with Actinium-255 PSMA617 RLT (activity $100 \mathrm{kBq} /$ kilogram bodyweight, applied at 8 -week intervals) showing promising results. Both patients had been extensively pretreated with at least 4 to 5 different mCRPC treatment regimens. One patient had shown progression upon treatment with Lu177 PSMA. Both patients showed a biochemical and radiographic complete remission following three cycles of Actinium-255 PSMA617. Although there was no severe hematologic toxicity observed, xerostomia occurred in both patients (25). One patient had severe xerostomia requiring permanent saliva subsitution via spray. A more recent, larger retrospective publication by Kratochwil et al. [2018] reported the application of up to three cycles of Actinium-255 PSMA617 at 8 -week intervals in $40 \mathrm{mCRPC}$ patients in late stage mCRPC who had exhausted conventional therapies including 7 patients who had prior treatment with Lu177PSMA (12). A total of $63 \%$ patients had a PSA decline $>50 \%$ with a median OS of $>12$ months and a median PFS of 7 months. Data collected at our institution at the Technical University Munich of 26 patients undergoing Ac255 PSMA for late stage mCRPC who had shown progression upon Lu177-PSMA treatment and had previously received at least second line antihormonal treatment and taxane based chemotherapy. Our data revealed a PSA decline $>50 \%$ in $65 \%$ of patients with a PSA PFS of 5 months and an OS of 7.7 months for salvage-treatment with Ac255-PSMA after progression under Lu177-PSMA. Of interest, Sathekge et al. investigated the use of Ac255-PSMA in 17 mCRPC chemotherapy-naïve patients and reported a PSA decline $>90 \%$ in $14 / 17$ patients, suggesting that Ac255-PSMA may be even more active at earlier disease stages (34).

However, to date, contrary to Lu177-PSMA, a prospective, randomized trial on Ac255-PSMA is not available. Reported retrospective data indicates that treatment with Actinium-255 PSMA-617 offers promising rates of tumor control. However, alpha emitter based RLT using Ac255-PSMA is associated with increased rates of xerostomia which may lead to discontinuation of treatment. Data from our workgroup showed that grade I-II xerostomia led to 6 patients $(33 \%)$ asking to discontinue treatment, whereas in the larger subset analyzed by Kratochwil et al. $10 \%$ of patients discontinued treatment due to intolerable xerostomia $(11,12)$. Strategies to overcome this limitation are eagerly needed. These may include reduction of treatment activity and tandem therapy using both Ac225-PSMA and Lu-177-PSMA simultaneously with lower activities of both radionuclides (35).

\section{Outlook on PSMA RLT}

Currently recruiting studies compare the safety, tolerability and efficacy of the combination of PSMA lutetium with the immune-checkpoint inhibitor pembrolizumab (e.g., phase I: NCT03658447, NCT03805594) or olaparib (e.g., phase I, NCT03874884) or as stand-alone treatment (e.g., phase II, NCT03454750, NCT04188587). The UpFrontPSMA phase II trial (NCT04343885) compares the safety and benefit of two inductive cycles of Lu-177-PSMA followed by six cycles of docetaxel versus six cycles of docetaxel alone patients with newly diagnosed high volume metastatic castration-sensitive PC. A non-randomized phase I/II trial (LuTectomy) investigates the dosimetry, efficacy and toxicity of two neoadjuvant cycles of Lu-177-PSMA in men with high-risk localized or locoregional advanced PC followed by radical prostatectomy (NCT04430192). The recently initiated open label, randomized, stratified, two-arm, multicenter phase II (ENZA-p) trial compares activity and safety of Lu-177-PSMA in combination with enzalutamide to enzalutamide alone in chemotherapy naïve mCRPC patients (NCT04419402). One prospective phase III trial (VISION study) is currently recruiting (NCT03511664).

Preliminary data of the randomized phase II (TheraP) trial presented at the ASCO2020 showed an improved biochemical response rate of Lu177-PSMA as compared to cabazitaxel in patients progressing upon docetaxel. The results of the prospective phase III VISION study investigating the efficacy of Lu177-PSMA in combination with standard of care versus best standard of care alone are eagerly anticipated.

The results of these, but also further prospective trials will help identify to role of Lu177-PSMA in comparison 
with approved treatment regimens and its application at an earlier time point in the treatment sequence of mCRPC. Moreover, additional trials are awaited to investigate the role of Lu-177-PSMA in combination with other drugs and its application in castration-sensitive PC, e.g., combination with standard of care for metastatic hormone-sensitive disease in the UpFrontPSMA trial and application in highrisk localized/locally advanced PC in the LuTectomy trial.

\section{Summary}

Available data suggests that RLT with Lu177-PSMA is an active therapy in late stage mCRPC and mostly well tolerated with a low side effect profile (xerostomia, fatigue or nausea). Severe, hematological side effects are rare. Data on treatment with Ac255-PSMA indicate a promising effect on tumor control in both early and late stage mCRPC. However, concerns are raised due to treatmentassociated and mostly permanent xerostomia, which seems to frequently lead to treatment discontinuation in a relevant number of patients.

Currently available, prospective phase II data on Lu177PSMA confirm previously published retrospective data. The results of ongoing phase II and phase III trials are eagerly anticipated to assess the efficacy of Lu177-PSMA in comparison with approved treatment regimens.

\section{Acknowledgments}

Funding: None.

\section{Footnote}

Provenance and Peer Review: This article was commissioned by the Guest Editor (Tilman Todenhöfer) for the series "Management of Advanced Genitourinary Malignancies" published in Translational Andrology and Urology. The article has undergone external peer review.

Reporting Checklist: The authors have completed the Narrative Review reporting checklist. Available at https:// dx.doi.org/10.21037/tau-20-1135

Peer Review File: Available at https://dx.doi.org/10.21037/ tau-20-1135

Conflicts of Interest: All authors have completed the ICMJE uniform disclosure form (available at https://dx.doi. org/10.21037/tau-20-1135). The series "Management of Advanced Genitourinary Malignancies" was commissioned by the editorial office without any funding or sponsorship. The authors have no other conflicts of interest to declare.

Ethical Statement: The authors are accountable for all aspects of the work in ensuring that questions related to the accuracy or integrity of any part of the work are appropriately investigated and resolved.

Open Access Statement: This is an Open Access article distributed in accordance with the Creative Commons Attribution-NonCommercial-NoDerivs 4.0 International License (CC BY-NC-ND 4.0), which permits the noncommercial replication and distribution of the article with the strict proviso that no changes or edits are made and the original work is properly cited (including links to both the formal publication through the relevant DOI and the license). See: https://creativecommons.org/licenses/by-nc-nd/4.0/.

\section{References}

1. Rahbar K, Ahmadzadehfar H, Kratochwil C, et al. German multicenter study investigating 177Lu-PSMA-617 Radioligand therapy in advanced prostate cancer patients. J Nucl Med 2017;58:85-90.

2. Silver DA, Pellicer I, Fair WR, et al. Prostate-specific membrane antigen expression in normal and malignant human tissues. Clin Cancer Res 1997;3:81-5.

3. Rajasekaran SA, Anilkumar G, Oshima E, et al. A novel cytoplasmic tail MXXXL motif mediates the internalization of prostate-specific membrane antigen. Mol Biol Cell 2003;14:4835-45.

4. Heck MM, Retz M, D’Alessandria C, et al. Systemic Radioligand Therapy with 177Lu Labeled Prostate Specific Membrane Antigen Ligand for Imaging and Therapy in Patients with Metastatic Castration Resistant Prostate Cancer. J Urol 2016;196:382-91.

5. Heck MM, Tauber R, Schwaiger S, et al. Treatment Outcome, Toxicity, and Predictive Factors for Radioligand Therapy with 177Lu-PSMA-I\&T in Metastatic Castration-resistant Prostate Cancer. Eur Urol 2019;75:920-6.

6. Benesová $M$, Schäfer $M$, Bauder-Wüst U, et al. Preclinical evaluation of a tailor-made DOTA-conjugated PSMA inhibitor with optimized linker moiety for imaging and endoradiotherapy of prostate cancer. J Nucl Med 2015;56:914-20. 
7. Kratochwil C, Giesel FL, Eder M, et al. $\left[{ }^{177} \mathrm{Lu}\right]$ Lutetiumlabelled PSMA ligand-induced remission in a patient with metastatic prostate cancer. Eur J Nucl Med Mol Imaging 2015;42:987-8.

8. Weineisen M, Schottelius M, Simecek J, et al. 68Ga- and 177Lu-Labeled PSMA I\&T: Optimization of a PSMATargeted Theranostic Concept and First Proof-of-Concept Human Studies. J Nucl Med 2015;56:1169-76.

9. Emami B, Lyman J, Brown A, et al. Tolerance of normal tissue to therapeutic irradiation. Int J Radiat Oncol Biol Phys 1991;21:109-22.

10. Fendler WP, Reinhardt S, Ilhan H, et al. Preliminary experience with dosimetry, response and patient reported outcome after 177Lu-PSMA-617 therapy for metastatic castration-resistant prostate cancer. Oncotarget 2017;8:3581-90.

11. Tauber R, Feuerecker B, Knorr K, et al. 872PSafety and efficacy of Ac-225-PSMA-617 in metastatic castration resistant prostate cancer (mCRPC) after failure of $\mathrm{Lu}-$ 177-PSMA. Ann Oncol 2019;30. doi: 10.1093/annonc/ $\operatorname{mdz} 248.029$.

12. Kratochwil C, Bruchertseifer F, Rathke H, et al. Targeted a-therapy of metastatic castration-resistant prostate cancer with 225 Ac-PSMA-617: Swimmer-Plot Analysis Suggests efficacy regarding duration of tumor control. J Nucl Med 2018;59:795-802.

13. Hofman MS, Violet J, Hicks RJ, et al. [177Lu]-PSMA-617 radionuclide treatment in patients with metastatic castration-resistant prostate cancer (LuPSMA trial): a single-centre, single-arm, phase 2 study. Lancet Oncol 2018;19:825-33.

14. Deutsche-Krebsgesellschaft, Deutsche-Krebshilfe A. Interdisziplinäre Leitlinie der Qualität S3 zur Früherkennung, Diagnose und Therapie der verschiedenen Stadien des Prostatakarzinoms. Available online: https:// www.leitlinienprogramm-onkologie.de/fileadmin/ user_upload/Downloads/Leitlinien/Prostata_5_0/LL_ Prostatakarzinom_Langversion_5.1.pdf

15. Kratochwil C, Fendler WP, Eiber M, et al. EANM procedure guidelines for radionuclide therapy with $177 \mathrm{Lu}-$ labelled PSMA-ligands (177Lu-PSMA-RLT). Eur J Nucl Med Mol Imaging 2019;46:2536-44.

16. Fendler W, Kratochwil C, Ahmadzadehfar H, et al. Therapie mit 177Lu-PSMA-617, Dosimetrie und Nachsorge beim metastasierten kastrationsresistenten Prostatakarzinom. Nuklearmedizin 2016;55:123-8.

17. Hofman MS, Emmett L, Violet J, et al. TheraP: a randomized phase 2 trial of 177 Lu-PSMA-617 theranostic treatment vs cabazitaxel in progressive metastatic castration-resistant prostate cancer (Clinical Trial Protocol ANZUP 1603). BJU Int 2019;124 Suppl 1:5-13.

18. Zechmann CM, Afshar-Oromieh A, Armor T, et al. Radiation dosimetry and first therapy results with a (124)I/ (131)I-labeled small molecule (MIP-1095) targeting PSMA for prostate cancer therapy. Eur J Nucl Med Mol Imaging 2014;41:1280-92.

19. Kratochwil C, Giesel FL, Stefanova M, et al. PSMAtargeted radionuclide therapy of metastatic castrationresistant prostate cancer with 177Lu-Labeled PSMA-617. J Nucl Med 2016;57:1170-6.

20. Ahmadzadehfar H, Eppard E, Kürpig S, et al. Therapeutic response and side effects of repeated radioligand therapy with 177Lu-PSMA-DKFZ-617 of castrate-resistant metastatic prostate cancer. Oncotarget 2016;7:12477-88.

21. Rahbar K, Schmidt M, Heinzel A, et al. Response and tolerability of a single dose of 177Lu-PSMA-617 in patients with metastatic castration-resistant prostate cancer: A multicenter retrospective analysis. J Nucl Med 2016;57:1334-8.

22. Violet J, Sandhu S, Iravani A, et al. Long-Term Followup and Outcomes of Retreatment in an Expanded 50-Patient Single-Center Phase II Prospective Trial of 177Lu-PSMA-617 Theranostics in Metastatic CastrationResistant Prostate Cancer. J Nucl Med 2020;61:857-65.

23. Baum RP, Kulkarni HR, Schuchardt C, et al. 177Lulabeled prostate-specific membrane antigen radioligand therapy of metastatic castration-resistant prostate cancer: Safety and efficacy. J Nucl Med 2016;57:1006-13.

24. Barber TW, Singh A, Kulkarni HR, et al. Clinical outcomes of 177Lu-PSMA radioligand therapy in earlier and later phases of metastatic castration-resistant prostate cancer grouped by previous taxane chemotherapy. J Nucl Med 2019;60:955-62.

25. Kratochwil C, Bruchertseifer F, Giesel FL, et al. 225AcPSMA-617 for PSMA-targeted a-radiation therapy of metastatic castration-resistant prostate cancer. J Nucl Med 2016;57:1941-4.

26. Sathekge M, Bruchertseifer F, Vorster M, et al. Predictors of overall and disease-free survival in metastatic castrationresistant prostate cancer patients receiving 225AcPSMA-617 radioligand therapy. J Nucl Med 2020;61:62-9.

27. Yadav MP, Ballal S, Sahoo RK, et al. Radioligand Therapy With 177Lu-PSMA for Metastatic Castration-Resistant Prostate Cancer: A Systematic Review and Meta-Analysis. AJR Am J Roentgenol 2019;213:275-85.

28. von Eyben FE, Roviello G, Kiljunen T, et al. Third- 
line treatment and 177Lu-PSMA radioligand therapy of metastatic castration-resistant prostate cancer: a systematic review. Eur J Nucl Med Mol Imaging 2018;45:496-508.

29. Calais J, Gartmann J, Armstrong WR, et al. Overall survival after 177 Lu-PSMA-617 molecular radiotherapy in patients with metastatic castrate-resistant prostate cancer: Post-hoc analysis of a prospective phase II trial. J Clin Oncol 2020;38:5549.

30. Vlachostergios PJ, Goswami S, Niaz MJ, et al. Patientreported outcomes (PRO) from a phase I/II doseescalation study of fractionated dose 177Lu-PSMA-617 for progressive metastatic castration-resistant prostate cancer (mCRPC). J Clin Oncol 2020;38:45.

31. Emmett L, Pathmanandavel S, Crumbaker M, et al. Updated results of a phase I/II prospective dose escalation trial evaluating safety and efficacy of combination 177 Lu PSMA 617 and idronoxil in men with mCRPC post androgen signalling inhibition and taxane chemotherapy

Cite this article as: Lunger L, Tauber R, Feuerecker B, Gschwend JE, Eiber M, Heck MM. Narrative review: prostatespecific membrane antigen-radioligand therapy in metastatic castration-resistant prostate cancer. Transl Androl Urol 2021;10(10):3963-3971. doi: 10.21037/tau-20-1135
(LuPIN trial). J Clin Oncol 2020;38:5557.

32. Gafita A, Rauscher I, Retz M, et al. Early experience of rechallenge 177Lu-PSMA radioligand therapy after an initial good response in patients with advanced prostate cancer. J Nucl Med 2019;60:644-8.

33. Emmett L, Willowson K, Violet J, et al. Lutetium 177 PSMA radionuclide therapy for men with prostate cancer: a review of the current literature and discussion of practical aspects of therapy. J Med Radiat Sci 2017;64:52-60.

34. Sathekge M, Bruchertseifer F, Knoesen O, et al. 225AcPSMA-617 in chemotherapy-naive patients with advanced prostate cancer: a pilot study. Eur J Nucl Med Mol Imaging 2019;46:129-38.

35. Khreish F, Ebert N, Ries M, et al. 225Ac-PSMA617/177Lu-PSMA-617 tandem therapy of metastatic castration-resistant prostate cancer: pilot experience. Eur J Nucl Med Mol Imaging 2020;47:721-8. 